P-83 ENHANCING BEREAVEMENT SUPPORT IN A TERTIARY CANCER CENTRE: A QUALITY IMPROVEMENT STUDY

Claire Cadwallader, Alison Coackley, Ann Griffiths, Amanda Watson, Julian HamptonMatthews, Malcom Cooper, Paula Horton. Clatterbridge Cancer Centre, CH63 4JY, UK

\subsection{6/bmjspcare-2017-00133.82}

Background Grief is a natural response to the loss of a loved one but can have the potential for long term adverse effects. Bereavement services are therefore essential to provide support and to identify those at risk of harm. A 'day after death service' for bereaved persons is a longstanding component of bereavement care at our tertiary cancer centre. Bereaved persons are met by a clinical nurse specialist the day after the death for a face to face discussion and support needs assessment. This system aims to improve support and identify those at risk of pathological grief reactions.

Aim Conduct a quality improvement project aimed at enhancing bereavement support at our centre by incorporating feedback from bereaved persons into trust-wide education and care quality indicators.

Methods A 12 month retrospective audit was undertaken to evaluate bereavement service outcomes.This was combined with feedback from stakeholders to develop a PDSA quality improvement cycle.

Results At baseline 51 deaths were recorded in 12 months. 42 people agreed to ongoing support following accessing the day after death service. 39 people did not require any further support following a single phone call. Three bereaved persons needed additional support due to prolonged/pathological grief, and were supported appropriately.

Stakeholder satisfaction surveys revealed an $88 \%$ approval rating for emotional support and practical help, and 100\% would recommend the service to others. Qualitative constructive feedback suggested enhancing methods of detecting people at high risk of pathological grief and developing an action-orientated approach to bereavement support.

Conclusion Bereavement care incurs practical as well as emotional challenges. Despite positive feedback regarding the emotional support provided by our service, there are practical areas for development. The next step of our project is to incorporate these changes into education and information processing tools, before re-evaluating progress.

\section{P-84 SUBJECTIVE AND OBJECTIVE CHEMOSENSORY (TASTE AND SMELL) DYSFUNCTION IN TREATMENT-NAIVE CANCER PATIENTS}

1,2 Michelle Barrett, ${ }^{1,3}$ Pauline U Dhuibhir, ${ }^{1,4}$ Niamh O'Donoghue ${ }^{5}$ Charles Gilham, ${ }^{5}$ Nazmi El Beltagi, ${ }^{1,4,6}$ Declan Walsh. 'Our Lady's Hospice and Care Services, Dublin 6, Ireland; ${ }^{2}$ School of Nursing and Midwifery, Trinity College Dublin, Ireland; ${ }^{3}$ School of Nursing, Midwifery and Health Science, University College Dublin, Ireland; ${ }^{4}$ School of Medicine, Trinity College Dublin, Ireland; ${ }^{5}$ St. Luke's Radiation Oncology Network, St James Hospital, Dublin, Ireland; ${ }^{6}$ School of Medicine and Medical Science, University College Dublin, Ireland

\subsection{6/bmjspcare-2017-00133.83}

Background Chemosensory dysfunction (CD; a defect in taste and/or smell) in cancer is associated with chemotherapy and radiotherapy or may occur as a result of cancer itself. CD can increase risk of weight loss and malnutrition as a result of food aversions and reduced dietary intake.
Aims

- To subjectively and objectively assess taste and smell in newly diagnosed non-head and neck cancer patients.

- To examine nutritional status in those with taste and smell changes.

Methods Consecutive pre-treatment cancer patients were recruited from radiation oncology clinics in a prospective observational study. A 12-item Taste and Smell Questionnaire evaluated the presence, severity and characteristics of taste and smell dysfunction and the Abridged Patient-Generated Subjective Global Assessment (abPG-SGA) measured nutritional status and symptoms. Burghart Taste Strips and 'Sniffen' Sticks Olfactory Test ${ }^{\circledast}$ assessed taste and smell identification.

Results Twenty newly diagnosed $(<3$ months) patients were recruited. Diagnoses included breast cancer (9) gynaecological cancer (7) and colorectal cancer (4). Over half $(n=12,60 \%)$ reported subjective taste and/or smell changes or had abnormal objective tests. Of these, six reported taste and smell changes (TSC), two taste only and one smell only. Nine were identified as being at risk of malnutrition (abPG-SGA score $\geq 6$ ). Four of them reported subjective taste or smell changes only while another two had both subjective and objective changes.

Conclusions Subjective and objective taste and smell testing were well tolerated. The majority of patients had subjective or objective TSC abnormalities before treatment. This is consistent with a previous study. Subjective assessment identified more TSC than objective. Those with TSC appeared to be at higher risk of malnutrition.

\section{P-85 THROMBOLYSIS FOR PULMONARY EMBOLISM IN PALLIATIVE CARE: A CASE REPORT}

${ }^{1}$ Mike Macfarlane, ${ }^{2}$ Louise Gilhooley, ${ }^{3}$ Tina Griffiths, ${ }^{1}$ Stephen Frost, ${ }^{2}$ Derek Willis. ${ }^{1}$ West Midlands Deanery, Telford, UK; ${ }^{2}$ Severn Hospice, Telford, UK; ${ }^{3}$ Shrewsbury and Telford Hospital NHS Trust, Telford, UK

\subsection{6/bmjspcare-2017-00133.84}

Background Thrombolysis for pulmonary embolism (PE) in a patient with advanced cancer has never been reported in the literature. Whilst not an absolute contraindication, most clinicians would be wary of thrombolysing a patient with a limited life expectancy. A case is presented of a patient with advanced mesothelioma who received thrombolysis treatment with a resultant significant improvement in pleuritic chest pain and dyspnoea.

History The patient was a 69 year old man who was diagnosed with epithelioid mesothelioma and received radiotherapy to his right chest followed by palliative chemotherapy. Subsequent CT scanning showed disease progression and the patient declined further chemotherapy.

Thrombolysis The patient was admitted to hospital with sudden-onset shortness of breath and right sided chest discomfort which was felt to be due to disease progression. Three days later the patient developed new, severe left sided pleuritic chest pain and acute breathlessness. The patient was thrombolysed with alteplase (50 mg IV over 1 hour) on clinical suspicion of a PE. The pleuritic chest pain resolved completely and the acute severe dyspnoea improved significantly within two hours of treatment and a CTPA later confirmed acute-onchronic pulmonary thromboembolism as well as progressive mesothelioma. The patient was transferred to the hospice four days later for ongoing management and end-of-life care. 